\title{
On the Performance of Beam Allocation Based Multi-User Massive MIMO Systems
}

\author{
Junyuan Wang*, Yuan $\mathrm{Kai}^{\dagger}$ and Huiling Zhu ${ }^{\dagger}$ \\ *Department of Computer Science, Edge Hill University, United Kingdom \\ ${ }^{\dagger}$ School of Engineering and Digital Arts, University of Kent, United Kingdom \\ Email: junyuan.wang@edgehill.ac.uk,yk69@kent.ac.uk, h.zhu@kent.ac.uk
}

\begin{abstract}
Moving to millimeter wave (mmWave) frequencies and deploying massive multiple input multiple output (MIMO) antenna arrays have shown great potential of supporting highdata-rate communications in the fifth-generation (5G) and beyond wireless networks, thanks to the availability of huge amounts of mmWave frequency bandwidth and massive numbers of narrow and high gain beams. A number of massive MIMO beamforming techniques have been proposed, among which the fixed-beam scheme has attracted considerable interests from both academia and industry due to its simplicity and requirement of a small number of radio frequency (RF) chains compared to the number of base-station (BS) antennas. Moreover, a beam allocation based pure analog fixed-beam system requires much lower complexity and less signalling overhead than the hybrid beamforming based fixed-beam system, which can therefore be easily implemented in the practical systems. In this paper, the sum data rate of beam allocation based multi-user massive MIMO systems is studied where a near-optimal low complexity beam allocation algorithm is adopted. Simulation results show that our derived average sum data rate serves as a good approximation of the simulation results.
\end{abstract}

Index Terms-Sum data rate analysis, downlink multi-user system, massive multiple-input-multiple-output (MIMO), beam allocation

\section{INTRODUCTION}

Massive multiple-input-multiple-output (MIMO) has been widely recognized as a promising technique for future wireless communication systems to meet the high-data-rate requirement in future use cases [1], [2]. By deploying a massive MIMO antenna array at the base-station (BS), narrow beams can be formed and high beam gains can be achieved [3], [4], which can compensate the severe propagation loss of mmWave signals and thus enables the use of millimeter wave (mmWave) spectrum. By integrating massive MIMO and mmWave communications technologies, system throughput can be further boosted as large amounts of bandwidth is available at mmWave frequencies.

Though with great potential of mmWave massive MIMO, the conventional digital beamforming techniques are difficult to be applied in practical massive MIMO systems as a signal needs to be independently fed into each BS antenna via a dedicated radio frequency (RF) chain, resulting in the requirement of a massive number of RF chains and thus unaffordable cost and extreamly high power consumption. In order to reduce the number of embedded RF chains, either forming a small number of beams in the analog domain at the RF end by adjusting the phase shifters connected to the BS antennas [5]-[10], or selecting some of beams from the preformed fixed analog beam set [11]-[15] was proposed for data transmission. By doing so, the massive MIMO antenna array can be connected to a small number of RF chains, which, fortunately, leads to little performance loss due to the spatial sparse property of mmWave channels [5]-[7].

With fixed beams, by applying hybrid analog-digital beamforming, selection of analog beams is first performed to decide the beams that will be activated and then digital beamforming is done in the beamspace of the selected analog beams to eliminate the mutual interference among beams [13]-[15]. Though with fixed analog beams, the instantaneous channel state information (CSI) [16]-[19] for all the channels between fixed beams and users are required to perform beam selection and baseband digital beamforming, which could bring huge signalling overhead to the system as the number of fixed beams is very large in a massive MIMO system for the sake of providing full coverage of the cell by narrow beams, and the number of users is increasing dramatically.

In order to reduce the signalling overhead, a simple beam allocation scheme for a fixed-beam system was proposed in [11] where a user is allocated a single dedicated beam for its data transmission and a near-optimal two-step low-complexity beam allocation (LBA) algorithm was proposed to maximize the system sum data rate. With this two-step LBA algorithm, each user is associated with its best beam with the highest beam gain among all the beams in the first step, and then in the second step, each associated beam is assigned to its best associated user with the highest received signal power [11]. It is clear from this algorithm that only the best beam gain for each user needs to be measured to apply the LBA algorithm, which leads to greatly reduced signalling overhead compared to fixed-beam based hybrid beamforming systems. As a result, such a beam allocation based scheme can be easily implemented in future massive MIMO systems where both the number of beams and the number of users are large. This motivates us to theoretically study the sum data rate performance of the beam allocation based multi-user massive MIMO system so as to provide direct guidance to future practical system design.

In this paper, a downlink beam allocation based multi-user massive MIMO system is considered where the BS is located at the center of a circular cell with $N$ fixed beams available for 


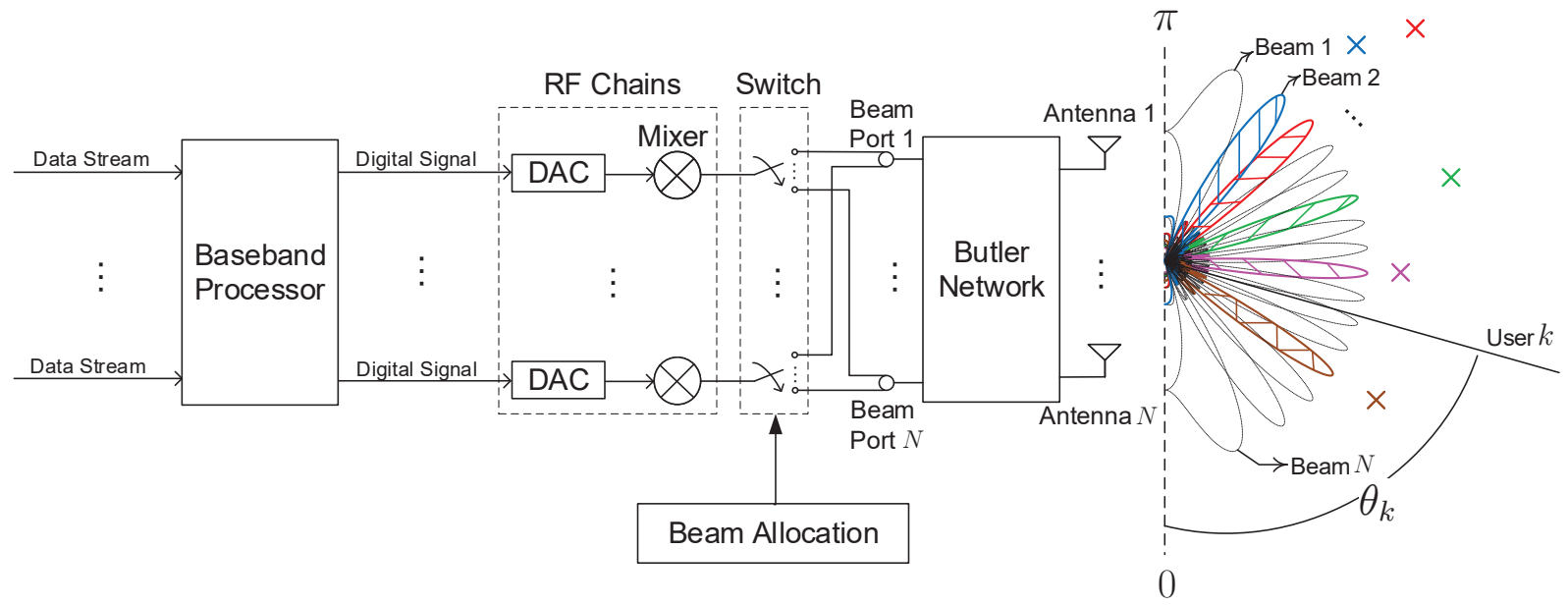

Fig. 1. Block diagram of a downlink fixed-beam based multi-user massive MIMO system with beam allocation. " $\mathrm{x}$ " represents a user. A user is allocated the beam in the same color.

data transmission, and $K$ users are randomly distributed in the cell. In order to study the sum data rate performance, the LBA algorithm proposed in [11] is employed in this paper. Since the fixed analog beams are directional, the beam gains seen by a user vary with its location. As a consequence, the beam allocation result closely depends on the user layout, leading to varying sum data rate as users move. Therefore, to study the sum data rate performance of the system, the average sum data rate over the locations of all the $K$ users, is studied in this paper. By ignoring the non-dominant interfering beams, the average sum data rate is derived as an explicit expression of the number of users $K$ and the number of beams $N$, which is verified by simulations.

The remainder of this paper is organized as follows. Section II introduces the system model and the beam allocation algorithm. The average sum data rate is analyzed in Section III, followed by the simulation results provided in Section IV. Finally, Section V concludes this paper.

Throughout this paper, $\mathbb{E}[\cdot]$ denotes the expectation operator. $|X|$ denotes the cardinality of set $X .\left(\begin{array}{l}n \\ k\end{array}\right)$ is a binomial coefficient denoting the number of ways to choose an unordered subset of $k$ elements form a fixed set of $n$ elements.

\section{System Model and BeAm Allocation}

The downlink transmission of a fixed-beam based multi-user massive MIMO system is considered as shown in Fig. 1. A fixed-beam base station (BS) antenna array is assumed to be located at the center of a circular cell, which consists of $N$ identical isotropic antenna elements that are linearly arranged and equally spaced at half wavelength of the propogation frequency. A Butler network [20] is assumed to be applied in the system at the RF end to form $N$ fixed beams. There are $K$ users uniformly distributed within the cell, each of which is equipped with a single antenna. The set of users is denoted by $\mathcal{K}$ with $|\mathcal{K}|=K$, and the set of fixed beams is denoted by $\mathcal{B}$ with $|\mathcal{B}|=N$. By applying the Butler network to form fixed beams, the directivity, i.e., beam gain, of any beam $n \in \mathcal{B}$ with respect to an angle of departure (AoD) $\theta$ is given in [11] as

$$
D_{n}(\theta)=\frac{\sin ^{2}\left(0.5 N \pi \cos \theta-\beta_{n}\right)}{N \sin ^{2}\left(0.5 \pi \cos \theta-\frac{1}{N} \beta_{n}\right)},
$$

with

$$
\beta_{n}=\left(-\frac{N+1}{2}+n\right) \pi .
$$

For any user $k \in \mathcal{K}$ located at $\mathbf{r}_{k}=\left(\rho_{k}, \theta_{k}\right)$, the AoD of the signal transmitted to user $k$ is $\theta_{k}$ as shown in Fig. 1, by assuming a line-of-sight (LoS) channel at millimeter-wave (mmWave) frequencies.

For a fixed-beam based multi-user massive MIMO system with beam allocation, the data for a user is tranmitted via its allocated beam. In a massive MIMO system, since the number of beams $N$ is much larger than the number of users $K$, only a number of beams will be turned on. Let $\mathcal{B}_{s}$ denote the set of active beams with $\left|\mathcal{B}_{s}\right|=N_{s}$. By performing a beam allocation algorithm, which user is allocated which beam is determined. Then the data signal of a user is fed into the corresponding beam port via a switch to activate its allocated beam for transmission, as illustrated in Fig. 1.

With the aim of maximizing the sum data rate of the massive MIMO system, a near-optimal low-complexity beam allocation (LBA) algorithm was developed in [11]. Specifically, by adopting the LBA algorithm, a user is firstly associated with its best beam with the highest directivity, and then an associated beam is allocated to its best user with the highest received signal power. Let beam $n_{k}^{(1)} \in \mathcal{B}_{s}$ be the beam allocated to user $k$. With the LBA algorithm, since a user $k$ is served by its best beam having the highest directivity, the index of the beam allocated to user $k, n_{k}^{(1)}$, is given by

$$
n_{k}^{(1)}=\arg \max _{n \in \mathcal{B}} D_{n}\left(\theta_{k}\right) \text {. }
$$


The power of the desired signal received at user $k$ is then obtained as

$$
P_{k}=p_{n_{k}^{(1)}} \cdot D_{n_{k}^{(1)}}\left(\theta_{k}\right) \cdot \rho_{k}^{-\alpha}
$$

where $p_{n_{k}^{(1)}}$ denotes the power allocated to beam $n_{k}^{(1)}$ for data transmission. $\rho_{k}$ is the access distance from user $k$ to the $\mathrm{BS}$, and $\alpha$ denotes the path-loss exponent. Let $P_{t}$ denote the total transmit power at the $\mathrm{BS}$, which is assumed to be equally allocated to the active beams. Then the transmit power on any beam $n_{k}^{(1)} \in \mathcal{B}_{s}$ is

$$
p_{n_{k}^{(1)}}=\frac{P_{t}}{N_{s}} .
$$

By assuming that the total system bandwidth is normalized to unity, the achievable sum data rate of the system can be presented as

$$
R_{s}=\sum_{n_{k}^{(1)} \in \mathcal{B}_{s}} R_{k}=\sum_{n_{k}^{(1)} \in \mathcal{B}_{s}} \log _{2}\left(1+\mu_{k}\right),
$$

where $R_{k}$ denotes the achievable data rate of users $k$ and

$$
\mu_{k}=\frac{P_{k}}{\sigma_{0}^{2}+I_{k}}
$$

denotes the received single-to-interference-plus-noise ratio (SINR) of user $k . \sigma_{0}^{2}$ denotes the variance of the additive white Gaussian noise (AWGN), i.e., noise power. $I_{k}$ denotes the power of user $k$ 's inter-beam interference, which can be easily obtained as

$$
I_{k}=\sum_{n_{j}^{(1)} \in \mathcal{B}_{s}, n_{j}^{(1)} \neq n_{k}^{(1)}} p_{n_{j}^{(1)}} \cdot D_{n_{j}^{(1)}}\left(\theta_{k}\right) \cdot \rho_{k}^{-\alpha} .
$$

It is obvious from (4) and (6)-(8) that the achievable sum data rate is determined by the beam allocation result which varies with the locations of users. In order to evaluate the performance from the system's persepctive, in this paper, we are interested in the average achievable sum data rate [21], [22], i.e., the achievable sum data rate averaged over all the possible locations of users, defined as

$$
\bar{R}_{s} \triangleq \mathbb{E}_{\left\{\mathbf{r}_{k}\right\}_{k \in \mathcal{K}}}\left[R_{s}\right]
$$

\section{Average Sum Data Rate Analysis}

In this section, the average sum data rate of beam allocation based massive MIMO systems will be analysed. Let us first have a look at the received SINR at user $k, \mu_{k}$.

\section{A. Received SINR $\mu_{k}$}

By combining (4)-(5) and (7)-(8), the received SINR $\mu_{k}$ for user $k$ can be obtained as

$$
\mu_{k}=\frac{\frac{P_{t}}{N_{s}} D_{n_{k}^{(1)}}\left(\theta_{k}\right) \rho_{k}^{-\alpha}}{\sigma_{0}^{2}+\sum_{n_{j}^{(1)} \in \mathcal{B}_{s}, n_{j}^{(1)} \neq n_{k}^{(1)}} \frac{P_{t}}{N_{s}} D_{n_{j}^{(1)}}\left(\theta_{k}\right) \rho_{k}^{-\alpha}} .
$$

Since the received SINR $\mu_{k}$ in (10) is a function of the directivities $\left\{D_{n_{j}^{(1)}}\left(\theta_{k}\right)\right\}_{n_{j}^{(1)} \in \mathcal{B}_{s}}$, the joint probability density function (pdf) of all the directivities $\left\{D_{n_{j}^{(1)}}\left(\theta_{k}\right)\right\}_{n_{j}^{(1)} \in \mathcal{B}_{s}}$ would be required to derive the average sum data rate, which, however, is very difficult to be obtained due to the randomness of users' locations. Therefore, an approximation will be derived in the following section to study the average sum data rate $\bar{R}_{s}$.

\section{B. Average Sum Data Rate $\bar{R}_{s}$}

In a beam allocation based massive MIMO system, a user's interference is mostly contributed by its strongest interfering beam similar to distributed antenna systems [23], [24]. Therefore, the SINR $\mu_{k}$ in (10) can be approximated by

$$
\mu_{k} \approx \mu_{k}^{a p}= \begin{cases}\frac{P_{t}}{N_{s} \sigma_{0}^{2}} D_{n_{k}^{(1)}}\left(\theta_{k}\right) \rho_{k}^{-\alpha}, & \text { if } N_{s}=1 ; \\ \frac{\frac{P_{t}}{N_{s}} D_{n_{k}^{(1)}}\left(\theta_{k}\right) \rho_{k}^{-\alpha}}{\sigma_{0}^{2}+\frac{P_{t}}{N_{s}} D_{n_{j}^{*}}\left(\theta_{k}\right) \rho_{k}^{-\alpha}}, & \text { if } \quad N_{s}>1,\end{cases}
$$

where

$$
n_{j}^{*}=\arg \max _{n_{j}^{(1)} \in \mathcal{B}_{s}, j \neq k} D_{n_{j}^{(1)}}\left(\theta_{k}\right)
$$

denotes the index of the strongest interfering beam of user $k$. The approximated average sum data rate can be then easily obtained by combining (6), (9) and (11) as

$$
\bar{R}_{s}=\mathbb{E}_{\left\{\mathbf{r}_{k}\right\}_{k \in \mathcal{K}}}\left[\sum_{n_{k}^{(1)} \in \mathcal{B}_{s}} \log _{2}\left(1+\mu_{k}^{a p}\right)\right] .
$$

Appendix A further presents that the average sum data rate $\bar{R}_{s}$ can be obtained as (14), which is shown at the bottom of this page, with

$$
n_{k}^{(l)}=\left\{\begin{array}{cl}
N+1-\frac{l}{2}, & \text { if } l \text { is even; } \\
\frac{l+1}{2}, & \text { if } l \text { is odd }
\end{array}\right.
$$

where $\left(\begin{array}{l}a \\ b\end{array}\right)=\frac{a !}{b !(a-b) !}$ denotes the binomial coefficient.

$\bar{R}_{s}=\sum_{m=2}^{K} m \cdot\left(\begin{array}{c}N \\ m\end{array}\right) \sum_{i=1}^{m}(-1)^{m-i}\left(\begin{array}{c}m \\ i\end{array}\right)\left(\frac{m}{N}\right)^{K} \sum_{l=2}^{N-m+2} \frac{\left(\begin{array}{c}N-l \\ m-2\end{array}\right)}{\left(\begin{array}{c}N-1 \\ m-1\end{array}\right)} \int_{0}^{1} \int_{\pi-\sqrt{\frac{2}{N}}}^{\pi} \log _{2}\left(1+\frac{\frac{P_{t}}{m} D_{1}(\theta) x^{-\alpha}}{\sigma_{0}^{2}+\frac{P_{t}}{m} D_{n_{k}(l)}(\theta) x^{-\alpha}}\right) \cdot \sqrt{\frac{N}{2}} \cdot 2 x d \theta d x$, 


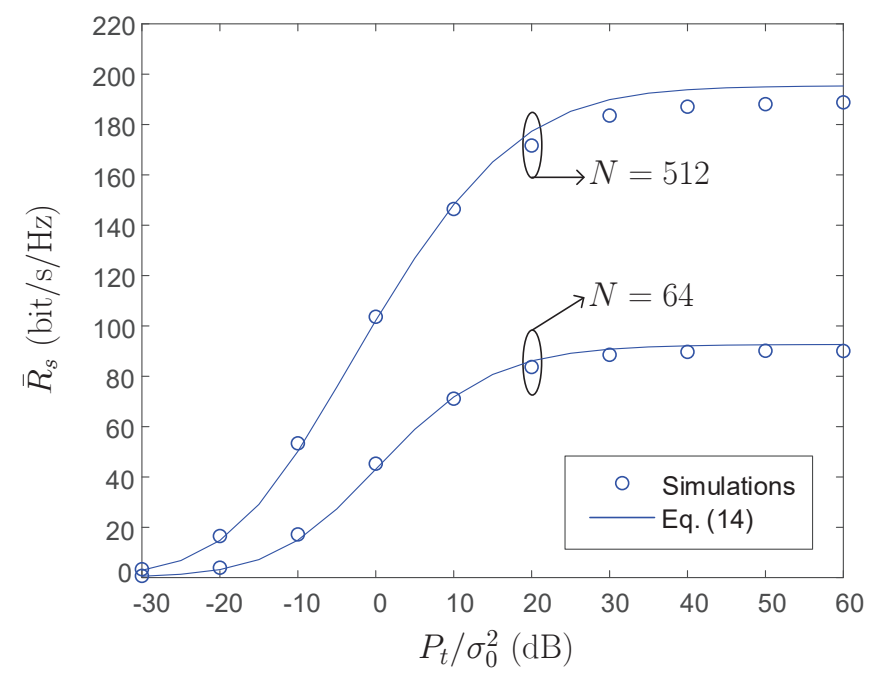

Fig. 2. Average sum data rate $\bar{R}_{s}$ versus the total transmit SNR $P_{t} / \sigma_{0}^{2}$. $\alpha=2.2, K=20, N=64,512$.

\section{Simulation Results}

Simulation results are presented in this section to demonstrate the effectiveness of the derived average sum data rate shown in (14). $K$ users are uniformly distributed within a circular cell with radius 1 . The simulated average sum data rate presented in this section is obtained by averaging over 10000 random realizations of the positions of $K$ users.

Fig. 2 presents the simulation results of the average sum data rate $\bar{R}_{s}$ versus the total transmit SNR $P_{t} / \sigma_{0}^{2}$ when the number of users $K$ is fixed at 20 and the number of beams $N=64,512$. Note that the average sum data rate $\bar{R}_{s}$ is derived in (14) as an explict function of system parameters, which is also plotted in the figure for the sake of comparison. We can see from Fig. 2 that (14) serves as a good approximation of the average sum data rate $\bar{R}_{s}$ regardless of the transmit SNR $P_{t} / \sigma_{0}^{2}$. As the number of beams $N$ increases from 64 to 512 , a very small rate gap can be observed when the transmit SNR $P_{t} / \sigma_{0}^{2}$ is high. This is because that the average sum data rate $\bar{R}_{s}$ is derived by neglecting the inter-beam interference from the non-dominant interfering beams. With a high $P_{t} / \sigma_{0}^{2}$, the system is interference-limited as shown in Fig. 2. For a larger number of beams $N$, there is a higher probablity that the interfering beams are far from the serving beam for a user, indicating that the variance of the interference powers received from different interfering beams becomes smaller. As a result, by only considering the dominant inter-beam interference, the numerical results of (14) are slightly higher than the simulation results.

To have a closer look at the effect of the total number of available beams $N$ on the effectiveness of the derived average sum data rate in (14), Fig. 3 further shows both the simulation results of the average sum data rate $\bar{R}_{s}$ and the numerical results of (14) with a varying number of beams $N$ when the transmit SNR $P_{t} / \sigma_{0}^{2}$ is $20 \mathrm{~dB}$ and there are $K=20$ users in the system. It is clearly shown in Fig. 3 that the analysis is

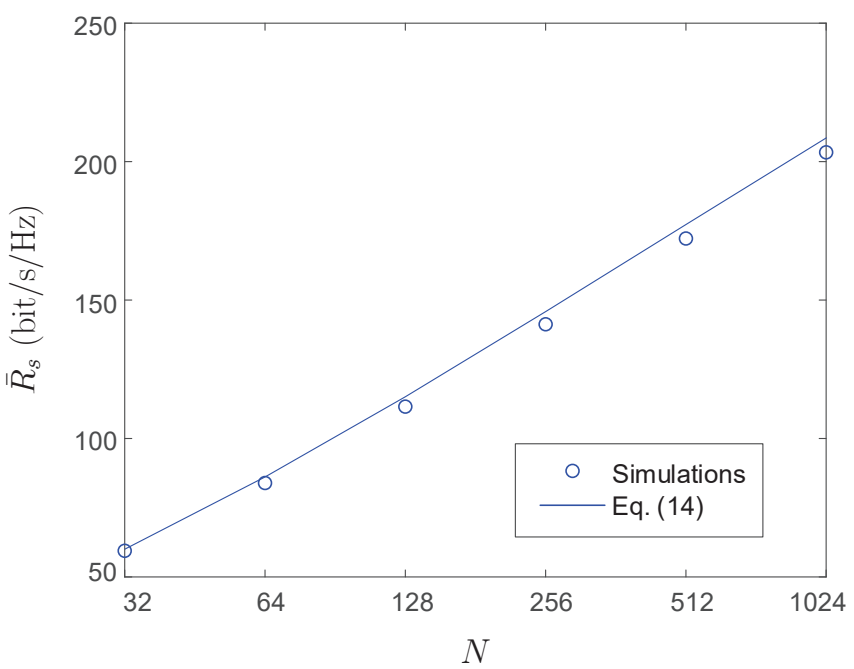

Fig. 3. Average sum data rate $\bar{R}_{s}$ versus the number of beams $N$. $\alpha=2.2$, $P_{t} / \sigma_{0}^{2}=20 \mathrm{~dB}, K=20$.

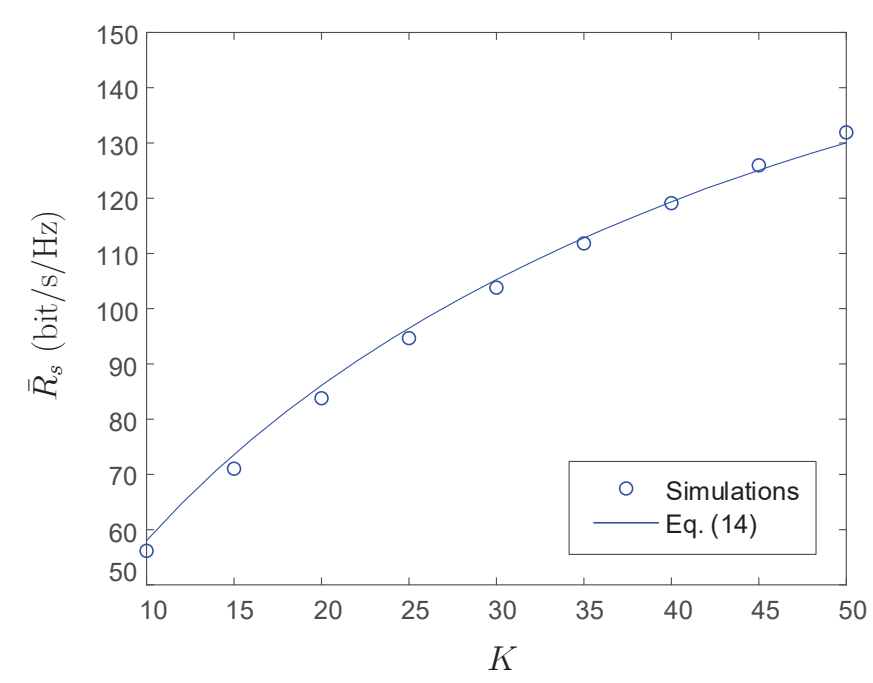

Fig. 4. Average achievable sum data rate $\bar{R}_{s}$ versus the number of users $K$. $\alpha=2.2, P_{t} / \sigma_{0}^{2}=20 \mathrm{~dB}, N=64$.

accurate as the number of beams $N$ increases from 32 to 1024 yet with a slightly increased gap between the analysis and the simulation results, as we expected from Fig. 2.

By fixing the number of beams $N$ at 64, Fig. 4 further shows the simulated average sum data rate $\bar{R}_{s}$ and its analytical results in (14) as the number of users $K$ increases from 10 to 50 . We can see from this figure that the average sum data rate can be well approximated by (14). Similar to Figs. 2 and 3 , the analysis is slightly higher if the number of beams $N$ is much larger than the number of users $K$. By contrast, when $K$ is comparable to $N,(14)$ is slightly lower than the simulation results due to the assumption adopted in the analysis that all the $N$ beams have the same beam width. 


\section{COnClusion}

This paper theorectically studied the sum data rate performance of a fixed-beam based multi-user massive MIMO system where beams are allocated to users for data transmission. As the sum data rate varies with users' positions, the sum date rate averaged over all the possible positions of users was analyzed. Specifically, the average sum data rate was derived as an explicit expression of the system parameters, i.e., the number of beams, the number of users and the available total transmit power, by only considering the strongest interfering beam and ignoring those which contribute less to the total inter-beam interference. Simulation results corroborate that the derived analytical expression provides a good approximation of average sum data rate.

\section{APPENDIX A}

DERIVATION OF (14) AND (15)

It is shown in (11) that the approximated received SINR at user $k, \mu_{k}^{a p}$, closely depends on the number of active beams $N_{s}$. By substituting (11) into (13), the average sum data rate $\bar{R}_{s}$ can be rewritten as

$$
\begin{aligned}
\bar{R}_{s}= & \mathbb{E}_{N_{s}}\left[\mathbb{E}_{\left\{\mathbf{r}_{k}\right\}_{k \in \mathcal{K}} \mid N_{s}}\left[\sum_{n_{k}^{(1)} \in \mathcal{B}_{s}} \log _{2}\left(1+\mu_{k}^{a p}\right) \mid N_{s}\right]\right] \\
= & \mathbb{E}_{\mathbf{r}_{k} \mid N_{s}=1}\left[\log _{2}\left(1+\mu_{k}^{a p} \mid N_{s}=1\right)\right] \cdot \operatorname{Pr}\left\{N_{s}=1\right\}+ \\
& \sum_{m=2}^{K} \mathbb{E}_{\left\{\mathbf{r}_{k}\right\}_{k \in \mathcal{K}} \mid N_{s}=m}\left[\sum_{n_{k}^{(1)} \in \mathcal{B}_{s}} \log _{2}\left(1+\mu_{k}^{a p}\right) \mid N_{s}=m\right] \\
& \cdot \operatorname{Pr}\left\{N_{s}=m\right\},
\end{aligned}
$$

where $\operatorname{Pr}\left\{N_{s}=m\right\}$ is the probability mass function (pmf) of the number of activated beams $N_{s}$. With $K$ users uniformly distributed within the cell and $N$ available fixed beams, the $\operatorname{pmf} \operatorname{Pr}\left\{N_{s}=m\right\}$ is given by [11]

$$
\operatorname{Pr}\left\{N_{s}=m\right\}=\left(\begin{array}{c}
N \\
m
\end{array}\right) \sum_{i=j}^{m}(-1)^{m-j}\left(\begin{array}{c}
m \\
j
\end{array}\right)\left(\frac{m}{N}\right)^{K},
$$

where $\left(\begin{array}{l}a \\ b\end{array}\right)=\frac{a !}{b !(a-b) !}$ denotes a binomial coefficient. By combining (A.1)-(A.2) and (11), we have

$$
\begin{aligned}
& \bar{R}_{s}^{u b} \stackrel{N}{\approx}^{1} \sum_{m=2}^{K} \operatorname{Pr}\left\{N_{s}=m\right\} . \\
& \mathbb{E}_{\left\{\mathbf{r}_{k}\right\}_{k \in \mathcal{K}} \mid N_{s}=m}\left[\sum_{n_{k}^{(1)} \in \mathcal{B}_{s}} \log _{2}\left(1+\frac{\frac{P_{t}}{m} D_{n_{k}^{(1)}}\left(\theta_{k}\right) \rho_{k}^{-\alpha}}{\sigma_{0}^{2}+\frac{P_{t}}{m} D_{n_{j}^{*}}\left(\theta_{k}\right) \rho_{k}^{-\alpha}}\right)\right] .
\end{aligned}
$$

For user $k$ located at $\mathbf{r}_{k}=\left(\rho_{k}, \theta_{k}\right)$, let

$$
D_{n_{k}^{(1)}}\left(\theta_{k}\right) \geq D_{n_{k}^{(2)}}\left(\theta_{k}\right) \cdots \geq D_{n_{k}^{(l)}}\left(\theta_{k}\right) \cdots \geq D_{n_{k}^{(N)}}\left(\theta_{k}\right)
$$

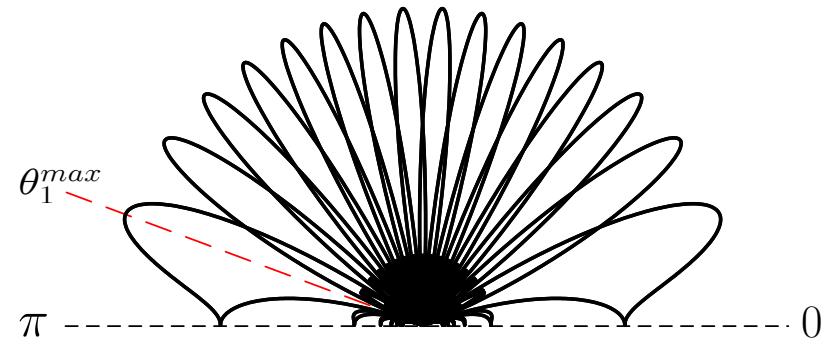

Fig. 5. Illustration of the angle of beam 1's main direction, $\theta_{1}^{\max }$.

denote the order statistics obtained by sorting the directivities $D_{1}\left(\theta_{k}\right), D_{2}\left(\theta_{k}\right), \cdots, D_{N}\left(\theta_{k}\right)$ of $N$ beams for user $k$, where $n_{k}^{(l)}$ denotes user $k$ 's $l$ th best beam with the $l$ th largest directivity. In particular, beam $n_{k}^{(1)}$ is allocated to user $k$ for its data transmission, and the index of the strongest interfering beam for user $k, n_{j}^{*}$, is given by

$$
n_{j}^{*}=n_{k}^{\left(l^{*}\right)}
$$

with

$$
l^{*}=\min _{n_{k}^{(l)} \in \mathcal{B}_{s}, l \neq 1} l .
$$

Given the number of active beams $N_{s}$, it is obvious that $2 \leq$ $l^{*} \leq N-N_{s}+2$. By forming a massive number of $N$ fixed beams using the Butler method, each beam approximately has identical shape. Without loss of generality, let us assume that beam 1 is allocated to the reference user $k$, i.e.,

$$
n_{k}^{(1)}=1,
$$

and is located within the angular range between the main direction of beam $1, \theta_{1}^{\max }$, and $\pi$ with

$$
\theta_{1}^{\max } \leq \theta_{k} \leq \pi,
$$

as illustrated in Fig. 5, where

$$
\theta_{1}^{\max }=\arg \max _{\theta} D_{1}(\theta) .
$$

By combining (1)-(2) and (A.9), it can be obtained that

$$
\theta_{1}^{\max }=\arccos \left(-1+\frac{1}{N}\right) \stackrel{N \gg 1}{\approx} \pi-\sqrt{\frac{2}{N}} .
$$

As $\theta_{1}^{\max } \leq \theta_{k} \leq \pi$, according to (A.7), we have

$$
n_{k}^{(l)}=\left\{\begin{array}{cl}
N+1-\frac{l}{2}, & \text { if } l \text { is even; } \\
\frac{l+1}{2}, & \text { if } l \text { is odd. }
\end{array}\right.
$$

By combining (A.3), (A.5)-(A.8) and (A.10), the average sum data rate $\bar{R}_{s}$ can be obtained as (A.12), shown at the top of the next page.

It is clear from (A.12) that the average sum rate $\bar{R}_{s}$ is a function of the pdfs of user $k$ 's polar coordinates, $f_{\rho_{k}}(x)$ and $f_{\theta_{k}}(\theta)$, and the conditional pmf of $l^{*}, \operatorname{Pr}\left\{l^{*}=l \mid N_{s}\right\}$, given the number of allocated beams $N_{s}$. As each beam has approximately identical width in a massive MIMO system where the number of beams is very large, each interfering user 
$\bar{R}_{s}^{u b}=\sum_{m=2}^{K} m \cdot \operatorname{Pr}\left\{N_{s}=m\right\} \sum_{l=2}^{N-m+2} \operatorname{Pr}\left\{l^{*}=l \mid N_{s}=m\right\} \int_{0}^{1} f_{\rho_{k}}(x) \int_{\pi-\sqrt{\frac{2}{N}}}^{\pi} f_{\theta_{k}}(\theta) \log _{2}\left(1+\frac{\frac{P_{t}}{m} D_{1}(\theta) x^{-\alpha}}{\sigma_{0}^{2}+\frac{P_{t}}{m} D_{n_{k}^{\left(l^{*}\right)}}(\theta) x^{-\alpha}}\right) d \theta d x$.

has the equal probability $1 /(N-1)$ to be allocated to anyone of the $N-1$ potential interfering beams. Therefore, given that the number of active beams is $N_{s}$, and beam $N / 2$ is already allocated to user $k$, the total number of combinations that $N_{s}$ out of $N$ beams are active is $\left(\begin{array}{c}N-1 \\ N_{s}-1\end{array}\right)$. Note that beam $n_{k}^{\left(l^{*}\right)}$ denotes the strongest interfering beam for user $k$, implying that beam $n_{k}^{\left(l^{*}\right)}$ is active and any beam $n_{k}^{(l)}$ with $1<l<l^{*}$ is off. In such a case, the number of combinations that $N_{s}$ out of $N$ beams are active is given by $\left(\begin{array}{c}N-l^{*} \\ N_{s}-2\end{array}\right)$. Therefore, given the number of active beams $N_{s}$, the corresponding conditional probability that user $k$ suffers from the strongest interference from beam $n_{k}^{\left(l^{*}\right)}$ can be obtained as

$$
\operatorname{Pr}\left\{l^{*}=l \mid N_{s}\right\}=\frac{\left(\begin{array}{c}
N-l \\
N_{s}-2
\end{array}\right)}{\left(\begin{array}{c}
N-1 \\
N_{s}-1
\end{array}\right)} .
$$

Since $K$ users are uniformly distributed within the cell with unit radius, the pdf of any user $k$ 's radial coordinate $\rho_{k}$ is given by

$$
f_{\rho}(x)=2 x,
$$

and the pdf of user $k$ 's angular coordinate $\theta_{k}$ is given by

$$
f_{\theta_{k}}(\theta)=\frac{1}{\pi-\theta_{1}^{\max }}
$$

due to $\theta_{1}^{\max } \leq \theta_{k} \leq \pi$. By subsituting (A.10) into (A.15), we have

$$
f_{\theta_{k}}(\theta)=\sqrt{\frac{N}{2}}
$$

Finally, by combining (A.2), (A.12)-(A.14) and (A.16), (14) can be obtained. (15) can be obtained according to (A.11).

\section{ACKNOWLEDGMENT}

Junyuan Wang would like to acknowledge the support of Edge Hill RIF Conference Travel Grant Award.

\section{REFERENCES}

[1] M. Shafi, A. F. Molisch, P. J. Smith, T. Haustein, P. Zhu, P. D. Silva, F. Tufvesson, A. Benjebbour, and G. Wunder, "5G: a tutorial overview of standards, trials, chanllenges, deployment, and practice," IEEE J. Select. Areas Commun., vol. 35, no. 6, pp. 1201-1221, June 2017.

[2] J. Wang, H. Zhu, and N. Gomes, "Distributed antenna systems for mobile communications in high speed trains," IEEE J. Sel. Areas Commun., vol. 30, pp. 675-683, May 2012.

[3] F. Rusek, D. Persson, B. K. Lau, E. G. Larsson, T. L. Marzetta, O. Edfors, and F. Tufvesson, "Scaling up MIMO: Opportunities and challenges with very large arrays," IEEE Signal Process. Mag., vol. 30, no. 1, pp. 40-60, Jan. 2013.

[4] M. Agiwal, A. Roy, and N. Saxena, "Next generation 5G wireless networks: a comprehensive survey," IEEE Commun. Sureys \& tutorials, vol. 18, no. 3, pp. 1617-1655, Third Quarter, 2016.
[5] O. E. Ayach, S. Rajagopal, S. Abu-Surra, Z. Pi, and R. W. Heath, "Spatially sparse precoding in millimeter wave MIMO systems," IEEE Trans. Wireless Commun., vol. 13, no. 3, pp. 1499-1513, Mar. 2014.

[6] T. E. Bogale, L. B. Le, A. Haghighat, and L. Vandendorpe, "On the number of RF chains and phase shifters, and scheduling design with hybrid analog-digital beamforming," IEEE Trans. Wireless Commun. vol. 15, no. 5, pp. 3311-3326, May 2016.

[7] M. N. Kulkarni, A. Ghosh, and J. G. Andrews, "A comparison of MIMO techniques in downlink millimeter wave cellular networks with hybrid beamforming," IEEE Trans. Commun., vol. 64, no. 5, pp. 1952-1967, May 2016.

[8] V. Venkateswaran and A. Veen, "Analog beamforming in MIMO communications with phase shift networks and online channel estimation," IEEE Trans. Signal Process., vol. 58, no. 8, pp. 4131-4143, Aug. 2010.

[9] S. Hur, T. Kim, D. J. Love, J. V. Krogmeier, T. A. Thomas, and A. Ghosh, "Millimeter wave beamforming for wireless backhaul and access in small cell networks," IEEE Trans. Commun., vol. 61, no. 10, pp. 4391-4403, Oct. 2013.

[10] V. V. Ratnam, A. F. Molisch, O. Y. Bursalioglu, and H. C. Papadopoulos, "Hybrid beamforming with selection for multi-user massive MIMO systems," in IEEE Trans. Signal Process., vol. 66, no. 15, pp. 41054120, Aug. 2018.

[11] J. Wang, H. Zhu, L. Dai, N. J. Gomes, and J. Wang, "Low-complexity beam allocation for switched-beam based multi-user massive MIMO systems," IEEE Trans. Wireless Commun., vol. 15, no. 12, pp. 82368248, Dec. 2016.

[12] J. Wang, H. Zhu, N. J. Gomes, and J. Wang, "Frequency reuse of beam allocation for multi-user massive MIMO systems," IEEE Trans. Wireless Commun., vol. 17, no. 4, pp. 2346-2359, Apr. 2018.

[13] J. Brady and A. Sayeed, "Beamspace MU-MIMO for high-density gigabit small cell access at millimeter-wave frequencies," in Proc. IEEE SPAWC, pp. 80-84, June 2014.

[14] P. Amadori and C. Masouros, "Low RF-complexity millimeter-wave beamspace-MIMO systems by beam selection," IEEE Trans. Wireless Commun., vol. 63, no. 6, pp. 2212-2223, June 2015.

[15] X. Gao, L. Dai, Z. Chen, Z. Wang, and Z. Zhang, "Near-optimal beam selection for beamspace mmWave massive MIMO systems," IEEE Commun. Letters, vol. 20, no. 5, pp. 1054-1057, May 2016.

[16] H. Zhu and J. Wang, "Chunk-based resource allocation in OFDMA systems - Part I: chunk allocation," IEEE Trans. Commun., vol. 57, no. 9, pp. 2734-2744, Sep. 2009.

[17] H. Zhu and J. Wang, "Chunk-based resource allocation in OFDMA systems - Part II: joint chunk, power and bit allocation," IEEE Trans. Commun., vol. 60, no. 2, pp. 499-509, Feb. 2012.

[18] H. Zhu and J. Wang, "Radio resource allocation in multiuser distributed antenna systems," IEEE J. Sel. Areas Commun., vol. 31, no. 10, pp. 2058-2066, Oct. 2013

[19] H. Zhu and J. Wang, "Performance analysis of chunk-based resource allocation in multi-cell OFDMA systems," IEEE J. Sel. Areas Commun., vol. 32, no. 2, pp. 367-375, Feb. 2014.

[20] J. Butler and R. Lowe, "Beam-forming matrix simplifies design of electrically scanned antennas," Electronic Design, Apr. 1962.

[21] H. Zhu, "Performance comparison between distributed antenna and microcellular systems," IEEE J. Sel. Areas Commun., vol. 29, pp. 11511163, 2011.

[22] H. Zhu, S. Karachontzitis, and D. Toumpakaris, "Low-complexity Resource allocation and its application to distributed antenna systems," IEEE Wireless Commun. Mag., vol. 17, no. 3, pp. 44-50, Jun. 2010.

[23] J. Wang and L. Dai, "Asymptotic rate analysis of downlink multi-user systems with co-located and distributed antennas," IEEE Trans. Wireless Commun., vol. 14, no. 6, pp. 3046-3058, Jun. 2015.

[24] J. Wang and L. Dai, "Downlink rate analysis for virtual-cell based largescale distributed antenna systems," IEEE Trans. Wireless Commun., vol. 15, no. 3, pp. 1998-2011, Mar. 2016. 